\title{
Ethnic heterogeneity and prostate cancer mortality in Hispanic/ Latino men: a population-based study
}

\author{
Felix M. Chinea ${ }^{1,2}$, Vivek N. Patel ${ }^{1,2}$, Deukwoo Kwon ${ }^{2}$, Narottam Lamichhane ${ }^{1,2}$, \\ Chris Lopez ${ }^{1,2}$, Sanoj Punnen ${ }^{2,3}$, Erin N. Kobetz ${ }^{2,4}$, Matthew C. Abramowitz ${ }^{1,2}$ and \\ Alan Pollack ${ }^{1,2}$ \\ ${ }^{1}$ Department of Radiation Oncology, University of Miami, Miami, FL, USA \\ ${ }^{2}$ Sylvester Comprehensive Cancer Center, University of Miami, Miami, FL, USA \\ ${ }^{3}$ Department of Urology, University of Miami, Miami, FL, USA \\ ${ }^{4}$ Division of Population Health and Computational Medicine, Department of Medicine, University of Miami, Miami, FL, USA \\ Correspondence to: Alan Pollack, email: apollack@med.miami.edu \\ Keywords: prostate cancer, cancer specific mortality, Hispanic/Latino, health disparities, minority health \\ Received: January 17, $2017 \quad$ Accepted: June 03, $2017 \quad$ Published: July 06, 2017 \\ Copyright: Chinea et al. This is an open-access article distributed under the terms of the Creative Commons Attribution License \\ 3.0 (CC BY 3.0), which permits unrestricted use, distribution, and reproduction in any medium, provided the original author and \\ source are credited.
}

\section{ABSTRACT}

Background: Few studies focus on prostate cancer (PCa) outcomes in Hispanic/ Latino men. Our study explores whether Hispanic/Latino subgroups demonstrate significantly different prostate cancer-specific mortality (PCSM) relative to NonHispanic White (NHW) and Non-Hispanic Black (NHB) men.

Methods: We extracted a population-based cohort of men diagnosed with localregional PCa from 2000-2013 $(n=486,865)$. PCSM was measured in racial/ethnic groups: NHW $(n=352,886)$, NHB $(n=70,983)$, Hispanic/Latino $(n=40,462)$, and Asian American/Pacific Islander $(n=22,534)$. PCSM was also measured in Hispanic/Latino subgroups: Mexican ( $n=8,077)$, Puerto Rican $(n=1,284)$, South or Central American $(n=3,021)$, Cuban $(n=788)$, and Dominican $(n=300)$. We conducted univariable and multivariable analyses (MVA) to compare risk for PCSM.

Results: Compared to NHW men, results showed worse outcomes for NHB men with similar outcomes for Hispanic/Latino men. In MVA with NHW men as a reference, NHB (HR= 1.15, $p<0.001)$ men had significantly worse PCSM and Hispanic/Latino ( $H R=1.02, p=0.534)$ men did not show a significant difference. In a second MVA, Puerto Rican (HR= 1.71, $p<0.001$ ) and Mexican $(H R=1.21, p=0.008)$ men had significantly higher PCSM. With NHB men as a reference, the MVA showed Puerto Rican (HR= 1.50, $p=0.006)$ men with higher PCSM and Mexican $(H R=1.08, p=0.307)$ men with no significant difference.

Conclusions: Our findings indicate previously unknown disparities in PCSM for Puerto Rican and Mexican American men.

\section{INTRODUCTION}

Disparities in prostate cancer $(\mathrm{PCa})$ treatment and outcome among Non-Hispanic Black (NHB) versus Non-Hispanic White (NHW) men have consistently been reported in the literature $[1,2]$. Socioeconomic and cultural factors, such as insurance coverage [2] or modifiable patient-provider decisions [3], contribute to such disparities. Unique biological differences have been reported as well $[4,5]$. Through careful consideration of potential influences of these disparities on disease incidence and outcomes, more exact conclusions can be made regarding the etiology. Currently, most inquiries focused on disparities fail to acknowledge important 
variability that occurs within racial and/or ethnic groups. Identifying such variability, should it exist, is essential for informing appropriate clinical screening and management of disease.

Individuals are typically assigned to a racial and/or ethnic group based on skin color [6], country of origin or ancestry, language or dialect spoken [7], and selfidentification. According to the U.S. Census Bureau, Hispanic or Latino individuals in 2014 reached about 55.4 million or $17.4 \%$ of the national population, making them the largest ethnic minority group in the United States of America [8]. Recent reports show that incidence and death rates among Hispanic/Latino men diagnosed with $\mathrm{PCa}$ are slightly lower than that of NHW men with NHB men having the highest rates [9]. As described in prior publications, there is the potential to over-generalize cancer outcome data by aggregating all Hispanic/Latino subgroups into one broad category for research purposes $[10,11]$.

Attempts to codify racial and/or ethnic categories will inevitably group heterogeneous populations. Within the Hispanic/Latino community, 64.9\% are Mexican, 9.2\% are Puerto Rican, $3.7 \%$ are Cuban, $3.0 \%$ are Dominican, and $19.2 \%$ are from other Hispanic/Latino groups [12]. With racial and/or ethnic categories being broad and overlapping, there is potential to obscure significant within-group differences $[7,10]$. Some studies have attempted to disaggregate ethnic subgroups by ancestry and/or geographic region of origin to highlight differences in health characteristics due to heterogeneity. A stroke study by Arauz et al [13] explored the difference in lipid profiles between a predominantly CaribbeanHispanic/Latino population in Miami, FL, U.S.A. and a predominantly Mestizo-Hispanic/Latino population in Mexico City, Mexico, ultimately finding significant differences in lipid profiles due to Hispanic/Latino racial or ethnic heterogeneity. Similarly, a nationwide populationbased study by Chao et al [14] features higher PCa-specific mortality (PCSM) rates in Hawaiian and Pacific Islander men compared to NHW and other Asian American subgroups. They describe the heterogeneity found within the Asian American/Pacific Islander (AAPI) group and that the results suggest the need for disaggregation of AAPI data. Potential underlying differences in PCSM amongst the genetically and culturally diverse Hispanic/ Latino subgroups have yet to be adequately characterized and then investigated for influential factors.

Despite those reporting survival differences based on cost of care [15], immigration status, and living in ethnic enclaves [16], much more is needed to further understand the potential role of ancestry and/or geographic region of origin in contribution to $\mathrm{PCa}$ incidence and outcome within the Hispanic/Latino male population. Recent studies examining racial and/or ethnic influences on $\mathrm{PCa}$, have focused on NHW and NHB men [17-19]. Among the few cancer-related articles discussing Hispanic/Latino subgroups, the use of state-specific cohorts may reduce their generalizability to the national population [20, 21]. To our knowledge, no other reports have addressed the heterogeneity within Hispanic/Latino men diagnosed with PCa from a national population-based cohort. Our study highlights the heterogeneity within Hispanic/Latino men and identifies populations that appear to be at greatest risk for mortality from PCa.

Our findings suggest that heterogeneity in PCSM exists within Hispanic/Latino men diagnosed with $\mathrm{PCa}$ in the United States. We identified two Hispanic/Latino subgroup populations with higher rates of PCSM after diagnosis with local-regional disease when compared to NHW men.

\section{RESULTS}

In Table 1, the baseline characteristics of the patient cohort divided into racial and/or ethnic groups (NHW, NHB, Hispanic/Latino, and AAPI) are displayed. Significant differences were found across these groups in marital status, insurance status, TNM stage, socioeconomic status (SES) composite score, disease stage summary, grade, residence type, treatment type, and age at diagnosis. In Table 2 we show the baseline characteristics for NHW men and all Hispanic/Latino ethnic subgroups. Although significant differences were found across these groups in TNM stage, SES composite score, grade, residence type, and treatment type ( $p$ $<0.001$ ); the magnitude of such differences were relatively small. Moreover, Mexican Americans were more likely than all other subgroups to have regional disease $(17.2 \%$ vs. 9.7-14.8\%) and use any Medicaid insurance (16.2\% vs. $8.2-14.3 \%$ ), while Puerto Ricans were less likely to be married (59.3\% vs. 65.3-73.9\%), and Cuban Americans were more likely to have a higher age at diagnosis (69.8 years vs. 64.6-65.9 years).

Figure 1 shows the cumulative incidence of PCSM among NHW, NHB, Hispanic/Latino, and AAPI men with a median follow-up of 50.4 months. NHB men have a higher PCSM than the Hispanic/Latino and NHW groups. Table 3 displays risk for PCSM in univariable analysis (UVA) and multivariable analysis (MVA) with NHW as the reference. In the UVA, NHB $(\mathrm{HR}=1.18,95 \%$ CI 1.12 to $1.24, p<0.001)$ men had significantly higher PCSM than NHW men; Hispanic/Latino (HR=1.07, 95\% CI 1.00 to $1.15, p=0.055)$ men did not show statistical significance and AAPI $(\mathrm{HR}=0.83,95 \%$ CI 0.75 to $0.92, p<0.001)$ men trended toward lower PCSM. This was reflected in the MVA with NHW men as the reference and adjusting for age at diagnosis, disease stage summary, grade, treatment type, SES composite score, insurance status, marital 
Table 1: Patient, tumor, and treatment characteristics divided by racial and/or ethnic group

\begin{tabular}{|c|c|c|c|c|c|c|}
\hline \multirow[t]{2}{*}{ Variable } & All & $\begin{array}{c}\text { Non-Hispanic } \\
\text { White }\end{array}$ & $\begin{array}{c}\text { Non-Hispanic } \\
\text { Black }\end{array}$ & $A A P I$ & Hispanic/Latino $^{a}$ & \multirow[t]{2}{*}{ p-value } \\
\hline & $N(\%)$ & $N(\%)$ & $N(\%)$ & $N(\%)$ & $N(\%)$ & \\
\hline Total patients & 486,865 & 352,886 & 70,983 & 22,534 & 40,462 & \\
\hline Age at diagnosis & $66.0 \pm 9.2$ & $66.5 \pm 9.2$ & $63.5 \pm 9.1$ & $67.9 \pm 9.0$ & $65.7 \pm 9.2$ & $<0.001$ \\
\hline Marital status & & & & & & $<0.001$ \\
\hline Married & $330,254(67.8)$ & $247,543(70.1)$ & $38,444(54.2)$ & $17,049(75.7)$ & $27,218(67.3)$ & \\
\hline Others & $102,160(21.0)$ & $66,177(18.8)$ & $24,292(34.2)$ & $3,141(13.9)$ & $8,550(21.1)$ & \\
\hline Unknown & $54,451(11.2)$ & $39,166(11.1)$ & 8,247 (11.6) & $2,344(10.4)$ & $4,694(11.6)$ & \\
\hline Insurance status & & & & & & $<0.001$ \\
\hline Uninsured & $4,745(1.0)$ & $2,224(0.6)$ & $1,463(2.1)$ & $207(0.9)$ & $851(2.1)$ & \\
\hline Any Medicaid & $14,770(3.0)$ & $5,521(1.6)$ & $3,809(5.4)$ & $1,893(8.4)$ & $3,547(8.8)$ & \\
\hline Insured & $322,916(66.3)$ & $238,642(67.6)$ & $46,172(65)$ & $13,721(60.9)$ & $24,381(60.3)$ & \\
\hline Unknown & $144,434(29.7)$ & $106,499(30.2)$ & $19,539(27.5)$ & $6,713(29.8)$ & $11,683(28.9)$ & \\
\hline T stage & & & & & & $<0.001$ \\
\hline$T x$ & $129(0.0)$ & $84(0.0)$ & $15(0.0)$ & $12(0.1)$ & $18(0.0)$ & \\
\hline TO & $5(0.0)$ & $3(0.0)$ & $1(0.0)$ & $0(0.0)$ & $1(0.0)$ & \\
\hline$T 1$ & $191,775(39.4)$ & $133,264(37.8)$ & $33,835(47.7)$ & $9,254(41.1)$ & $15,422(38.1)$ & \\
\hline$T 2$ & $248,490(51.0)$ & $185,184(52.5)$ & $31,546(44.4)$ & $10,841(48.1)$ & $20,919(51.7)$ & \\
\hline$T 3$ & $41,830(8.6)$ & $31,110(8.8)$ & 4,924 (6.9) & $2,166(9.6)$ & $3,630(9.0)$ & \\
\hline$T 4$ & $4,636(1.0)$ & $3241(0.9)$ & $662(0.9)$ & $261(1.2)$ & $472(1.2)$ & \\
\hline N stage & & & & & & $<0.001$ \\
\hline$N x$ & $5,659(1.2)$ & $3,960(1.1)$ & $778(1.1)$ & $335(1.5)$ & $586(1.4)$ & \\
\hline NO & $474,280(97.4)$ & $343,954(97.5)$ & $69,250(97.6)$ & $21,890(97.1)$ & $39,186(96.8)$ & \\
\hline$N 1$ & $6,926(1.4)$ & 4972 (1.4) & $955(1.3)$ & $309(1.4)$ & $690(1.7)$ & \\
\hline M stage & & & & & & $<0.001$ \\
\hline MO & $486,865(100)$ & $352,886(100)$ & $70,983(100)$ & $22,534(100)$ & $40,462(100)$ & \\
\hline SES composite sc & ore & & & & & $<0.001$ \\
\hline 1 & $100,029(20.5)$ & $78,132(22.1)$ & $8,122(11.4)$ & $8,491(37.7)$ & $5,284(13.1)$ & \\
\hline 2 & $99,427(20.4)$ & $78,701(22.3)$ & $9,481(13.4)$ & $5,201(23.1)$ & 6,044 (14.9) & \\
\hline 3 & $93,504(19.2)$ & $74,423(21.1)$ & $11,259(15.9)$ & $2,475(11.0)$ & $5,347(13.2)$ & \\
\hline 4 & $109,772(22.5)$ & $66,596(18.9)$ & $20,776(29.3)$ & $5,283(23.4)$ & $17,117(42.3)$ & \\
\hline 5 & $84,005(17.3)$ & $54,947(15.6)$ & $21,340(30.1)$ & $1,083(4.8)$ & $6,635(16.4)$ & \\
\hline Unknown & $128(0.0)$ & $87(0.0)$ & $5(0.0)$ & $1(0.0)$ & $35(0.1)$ & \\
\hline Stage summary & & & & & & $<0.001$ \\
\hline Localized & $423,231(86.9)$ & 306,113 (86.7) & $63,134(88.9)$ & $19,255(85.4)$ & $34,729(85.8)$ & \\
\hline Regional & $63,634(13.1)$ & $46,773(13.3)$ & $7,849(11.1)$ & $3,279(14.6)$ & $5,733(14.2)$ & \\
\hline
\end{tabular}

(Continued) 


\begin{tabular}{|c|c|c|c|c|c|c|}
\hline \multirow[t]{2}{*}{ Variable } & All & $\begin{array}{c}\text { Non-Hispanic } \\
\text { White }\end{array}$ & $\begin{array}{c}\text { Non-Hispanic } \\
\text { Black }\end{array}$ & $A A P I$ & Hispanic/Latino $^{a}$ & \multirow[t]{2}{*}{ p-value } \\
\hline & $N(\%)$ & $N(\%)$ & $N(\%)$ & $N(\%)$ & $N(\%)$ & \\
\hline Residence type & & & & & & $<0.001$ \\
\hline Rural & $6,316(1.3)$ & $5,691(1.6)$ & $537(0.8)$ & $4(0.0)$ & $84(0.2)$ & \\
\hline Urban & $480,421(98.7)$ & $347,108(98.4)$ & $70,441(99.2)$ & $22,529(100)$ & $40,343(99.7)$ & \\
\hline Unknown & $128(0.0)$ & $87(0.0)$ & $5(0.0)$ & $1(0.0)$ & $35(0.1)$ & \\
\hline Treatment type & & & & & & $<0.001$ \\
\hline None & 106,567 (21.9) & $74,650(21.2)$ & $17,597(24.8)$ & 4,993 (22.2) & 9,327 (23.1) & \\
\hline RT only & $162,646(33.4)$ & $115503(32.7)$ & $26,613(37.5)$ & $7,960(35.3)$ & $12,570(31.1)$ & \\
\hline Surgery only & 194,237 (39.9) & $145,939(41.4)$ & $23,297(32.8)$ & $8,358(37.1)$ & $16,643(41.1)$ & \\
\hline Surgery $+R T$ & $13,230(2.7)$ & $9,405(2.7)$ & $1,837(2.6)$ & $763(3.4)$ & $1,225(3.0)$ & \\
\hline $\begin{array}{l}\text { Surgery }+ \\
\text { Unknown RT }\end{array}$ & $2,681(0.6)$ & 2,098 (0.6) & $324(0.5)$ & $107(0.5)$ & $152(0.4)$ & \\
\hline Unknown & $7,249(1.5)$ & $5,093(1.4)$ & $1,272(1.8)$ & $349(1.5)$ & $535(1.3)$ & \\
\hline $\begin{array}{l}\text { Unknown } \\
\text { Surgery }+R T\end{array}$ & $255(0.1)$ & $198(0.1)$ & $43(0.1)$ & $4(0.0)$ & $10(0.0)$ & \\
\hline \multicolumn{7}{|l|}{ Grade $^{\mathrm{b}}$} \\
\hline $\begin{array}{l}\text { Well } \\
\text { differentiated }\end{array}$ & $4,926(1.0)$ & $3,510(1.0)$ & $626(0.9)$ & $229(1.0)$ & $561(1.4)$ & $<0.001$ \\
\hline $\begin{array}{l}\text { Moderately } \\
\text { differentiated }\end{array}$ & $216,986(44.6)$ & $159,535(45.2)$ & $29,704(41.8)$ & 8,969 (39.8) & $18,778(46.4)$ & \\
\hline $\begin{array}{l}\text { Poorly } \\
\text { differentiated }\end{array}$ & $252,492(51.9)$ & $181,012(51.3)$ & $38,726(54.6)$ & $12,732(56.5)$ & $20,022(49.5)$ & \\
\hline Undifferentiated & $1,044(0.2)$ & $732(0.2)$ & $181(0.3)$ & $46(0.2)$ & $85(0.2)$ & \\
\hline Unknown & $11,417(2.3)$ & 8097 (2.3) & $1,746(2.5)$ & $558(2.5)$ & $1,016(2.5)$ & \\
\hline
\end{tabular}

Abbreviations: AAPI = Asian American/Pacific Islander; SES = socioeconomic status; RT = radiotherapy.

P-values were obtained from Kruskal-Wallis test for continuous variable and Chi-square test for categorical variables.

${ }^{a}$ Represents all Hispanic/Latino individuals from the study, including those reported as Hispanic/Latino, NOS.

${ }^{b}$ Well differentiated, moderately differentiated, and poorly differentiated histologic grading correlate with Gleason scores $2-4,5-6$, and 7-10, respectively.

status, and residence type. NHB (HR=1.15, 95\% CI 1.09 to $1.22, p<0.001)$ men had significantly worse PCSM and AAPI (HR $=0.74,95 \%$ CI 0.67 to $0.82, p<0.001)$ men had improved PCSM, while Hispanic/Latino (HR=1.02, $95 \%$ CI 0.95 to $1.10, p=0.534$ ) men showed no statistical significance.

Disaggregation of the Hispanic/Latino cohort into ethnic subgroups is shown in Figure 1. Puerto Rican, Mexican, and Cuban groups had higher PCSM than not only NHW and AAPI men, but NHB men as well. The UVAs with NHW as the reference are displayed in Table 3 , showing that Mexican (HR $=1.34,95 \%$ CI 1.17 to 1.53 , $p<0.001)$ and Puerto Rican $(\mathrm{HR}=1.70,95 \%$ CI 1.28 to
2.25, $p<0.001)$ men had significantly higher PCSM. In the MVA with NHW as the reference, Mexican ( $\mathrm{HR}=1.21$, $95 \%$ CI 1.05 to $1.39, p=0.008)$ and Puerto Rican $(\mathrm{HR}=$ $1.71,95 \%$ CI 1.29 to $2.26, p<0.001)$ men had significantly higher PCSM compared to the NHW cohort.

In order to directly compare Hispanic/Latino subgroups at high risk for PCSM with NHB men, the most at-risk population identified thus far, we conducted additional regression models with NHB men as a reference. In the UVAs (Table 4), Puerto Rican $(\mathrm{HR}=1.45,95 \% \mathrm{CI} 1.09$ to $1.92, p=0.011)$ men had significantly higher PCSM compared to NHB men and Mexican (HR=1.14, 95\% CI 0.99 to $1.31, p=0.065)$ men 
Table 2: Patient, tumor, and treatment characteristics divided by racial and/or ethnic subgroup

\begin{tabular}{|c|c|c|c|c|c|c|c|c|}
\hline \multirow[t]{2}{*}{ Variable } & All & $\begin{array}{c}\text { Non- } \\
\text { Hispanic } \\
\text { White }\end{array}$ & Mexican & Cuban & $\begin{array}{c}\text { Puerto } \\
\text { Rican }\end{array}$ & $\begin{array}{c}\text { Dominican } \\
\text { Republic }\end{array}$ & $\begin{array}{c}\text { South or } \\
\text { Central } \\
\text { American }\end{array}$ & \multirow[t]{2}{*}{ p-value } \\
\hline & $N(\%)$ & $N(\%)$ & $N(\%)$ & $N(\%)$ & $N(\%)$ & $N(\%)$ & $N(\%)$ & \\
\hline Total patients & 366,356 & 352,886 & 8,077 & 788 & 1,284 & 300 & 3,021 & \\
\hline Age at diagnosis & $66.4 \pm 9.2$ & $66.5 \pm 9.2$ & $65.9 \pm 9.0$ & $69.8 \pm 8.9$ & $65.8 \pm 9.2$ & $64.6 \pm 9.0$ & $65.0 \pm 8.9$ & $<0.001$ \\
\hline Marital status & & & & & & & & $<0.001$ \\
\hline Married & $\begin{array}{c}257,161 \\
(70.2)\end{array}$ & $\begin{array}{c}247,543 \\
(70.1)\end{array}$ & $5,971(73.9)$ & $531(67.4)$ & $761(59.3)$ & $196(65.3)$ & $\begin{array}{l}2,159 \\
(71.5)\end{array}$ & \\
\hline Others & 69,078 (18.9) & $66,177(18.8)$ & $1,534(19.0)$ & $209(26.5)$ & $421(32.8)$ & $71(23.7)$ & $666(22.0)$ & \\
\hline Unknown & $40,117(11.0)$ & $39,166(11.1)$ & $572(7.1)$ & $48(6.1)$ & $102(7.9)$ & $33(11.0)$ & $196(6.5)$ & \\
\hline Insurance status & & & & & & & & $<0.001$ \\
\hline Uninsured & $2,656(0.7)$ & $2,224(0.6)$ & $234(2.9)$ & $8(1.0)$ & $29(2.3)$ & $14(4.7)$ & $147(4.9)$ & \\
\hline Any Medicaid & $7,404(2.0)$ & $5,521(1.6)$ & $1,305(16.2)$ & $65(8.2)$ & $117(9.1)$ & $43(14.3)$ & $353(11.7)$ & \\
\hline Insured & $\begin{array}{c}245,597 \\
(67.0)\end{array}$ & $\begin{array}{c}238,642 \\
(67.6)\end{array}$ & $4,069(50.4)$ & $423(53.7)$ & $712(55.5)$ & $174(58.0)$ & $\begin{array}{l}1,577 \\
(52.2)\end{array}$ & \\
\hline Unknown & $\begin{array}{c}110,699 \\
(30.2)\end{array}$ & $\begin{array}{c}106,499 \\
(30.2)\end{array}$ & $2,469(30.6)$ & $292(37.1)$ & $426(33.2)$ & $69(23.0)$ & 944 (31.2) & \\
\hline T stage & & & & & & & & $<0.001$ \\
\hline$T x$ & $90(0.0)$ & $84(0.0)$ & $4(0.0)$ & $2(0.3)$ & $0(0.0)$ & $0(0.0)$ & $0(0.0)$ & \\
\hline TO & $3(0.0)$ & $3(0.0)$ & $0(0.0)$ & $0(0.0)$ & $0(0.0)$ & $0(0.0)$ & $0(0.0)$ & \\
\hline$T 1$ & $\begin{array}{c}138,398 \\
(37.8)\end{array}$ & $\begin{array}{c}133,264 \\
(37.8)\end{array}$ & $2,873(35.6)$ & $368(46.7)$ & $576(44.9)$ & $155(51.7)$ & $\begin{array}{l}1,162 \\
(38.5)\end{array}$ & \\
\hline$T 2$ & $\begin{array}{c}192,010 \\
(52.4)\end{array}$ & $\begin{array}{c}185,184 \\
(52.5)\end{array}$ & $4,244(52.5)$ & $356(45.2)$ & $570(44.4)$ & $124(41.3)$ & $\begin{array}{l}1,532 \\
(50.7)\end{array}$ & \\
\hline$T 3$ & $32,436(8.9)$ & $31,110(8.8)$ & $836(10.4)$ & $52(6.6)$ & $122(9.5)$ & $17(5.7)$ & $299(9.9)$ & \\
\hline T4 & $3,419(0.9)$ & $3,241(0.9)$ & $120(1.5)$ & $10(1.3)$ & $16(1.2)$ & $4(1.3)$ & $28(0.9)$ & \\
\hline N stage & & & & & & & & $<0.001$ \\
\hline$N x$ & $4,194(1.1)$ & $3,960(1.1)$ & $124(1.5)$ & $22(2.8)$ & 25 (1.9) & $12(4)$ & $51(1.7)$ & \\
\hline NO & $\begin{array}{c}356,898 \\
(97.4)\end{array}$ & $\begin{array}{c}343,954 \\
(97.5)\end{array}$ & $7,763(96.1)$ & $755(95.8)$ & $\begin{array}{l}1,240 \\
(96.6)\end{array}$ & $281(93.7)$ & $\begin{array}{l}2,905 \\
(96.2)\end{array}$ & \\
\hline$N 1$ & $5,264(1.4)$ & $4,972(1.4)$ & $190(2.4)$ & $11(1.4)$ & $19(1.5)$ & $7(2.3)$ & $65(2.2)$ & \\
\hline M stage & & & & & & & & $<0.001$ \\
\hline MO & $\begin{array}{c}366,356 \\
(100)\end{array}$ & $\begin{array}{c}352,886 \\
(100)\end{array}$ & $8,077(100)$ & $788(100)$ & $\begin{array}{l}1,284 \\
(100)\end{array}$ & $300(100)$ & $3,021(100)$ & \\
\hline SES composite sc & ore & & & & & & & $<0.001$ \\
\hline 1 & $79,948(21.8)$ & $78,132(22.1)$ & $731(9.1)$ & $117(14.8)$ & $366(28.5)$ & $77(25.7)$ & $525(17.4)$ & \\
\hline 2 & $80,492(22.0)$ & $78,701(22.3)$ & $826(10.2)$ & $134(17.0)$ & $299(23.3)$ & $59(19.7)$ & $473(15.7)$ & \\
\hline 3 & $76,149(20.8)$ & $74,423(21.1)$ & $1,153(14.3)$ & $58(7.4)$ & $205(16.0)$ & $43(14.3)$ & $267(8.8)$ & \\
\hline 4 & $73,218(20.0)$ & $66,596(18.9)$ & $4052(50.2)$ & $462(58.6)$ & $315(24.5)$ & $112(37.3)$ & $\begin{array}{l}1,681 \\
(55.6)\end{array}$ & \\
\hline
\end{tabular}

(Continued) 


\begin{tabular}{|c|c|c|c|c|c|c|c|c|}
\hline \multirow[t]{2}{*}{ Variable } & All & $\begin{array}{c}\text { Non- } \\
\text { Hispanic } \\
\text { White }\end{array}$ & Mexican & Cuban & $\begin{array}{l}\text { Puerto } \\
\text { Rican }\end{array}$ & $\begin{array}{c}\text { Dominican } \\
\text { Republic }\end{array}$ & $\begin{array}{c}\text { South or } \\
\text { Central } \\
\text { American }\end{array}$ & \multirow[t]{2}{*}{ p-value } \\
\hline & $N(\%)$ & $N(\%)$ & $N(\%)$ & $N(\%)$ & $N(\%)$ & $N(\%)$ & $N(\%)$ & \\
\hline 5 & $56,460(15.4)$ & $54,947(15.6)$ & $1,315(16.3)$ & $16(2.0)$ & $98(7.6)$ & $9(3.0)$ & $75(2.5)$ & \\
\hline Unknown & $89(0.0)$ & $87(0.0)$ & $0(0.0)$ & $1(0.1)$ & $1(0.1)$ & $0(0.0)$ & $0(0.0)$ & \\
\hline Stage summary & & & & & & & & $<0.001$ \\
\hline Localized & $\begin{array}{c}317,454 \\
(86.7)\end{array}$ & $\begin{array}{c}306,113 \\
(86.7)\end{array}$ & $6,691(82.8)$ & $703(89.2)$ & $\begin{array}{l}1,103 \\
(85.9)\end{array}$ & $271(90.3)$ & $\begin{array}{l}2,573 \\
(85.2)\end{array}$ & \\
\hline Regional & $48,902(13.3)$ & $46,773(13.3)$ & $1,386(17.2)$ & $85(10.8)$ & $181(14.1)$ & $29(9.7)$ & $448(14.8)$ & \\
\hline Residence type & & & & & & & & $<0.001$ \\
\hline Rural & $5,704(1.6)$ & $5,691(1.6)$ & $12(0.1)$ & $0(0.0)$ & $1(0.1)$ & $0(0.0)$ & $0(0.0)$ & \\
\hline Urban & $\begin{array}{c}360,563 \\
(98.4)\end{array}$ & $\begin{array}{c}347,108 \\
(98.4)\end{array}$ & 8,065 (99.9) & 787 (99.9) & $\begin{array}{l}1,282 \\
(99.8)\end{array}$ & $300(100)$ & $3,021(100)$ & \\
\hline Unknown & $89(0.0)$ & $87(0.0)$ & $0(0.0)$ & $1(0.1)$ & $1(0.1)$ & $0(0.0)$ & $0(0.0)$ & \\
\hline Treatment type & & & & & & & & $<0.001$ \\
\hline None & $77,324(21.1)$ & $74,650(21.2)$ & $1,680(20.8)$ & $144(18.3)$ & $227(17.7)$ & $48(16.0)$ & $575(19.0)$ & \\
\hline RT only & $\begin{array}{c}119,944 \\
(32.7)\end{array}$ & $\begin{array}{c}115,503 \\
(32.7)\end{array}$ & $2,439(30.2)$ & $366(46.4)$ & $553(43.1)$ & $132(44.0)$ & $951(31.5)$ & \\
\hline Surgery only & $\begin{array}{c}151,569 \\
(41.4)\end{array}$ & $\begin{array}{c}145,939 \\
(41.4)\end{array}$ & $3,544(43.9)$ & $238(30.2)$ & $429(33.4)$ & $96(32.0)$ & $\begin{array}{l}1,323 \\
(43.8)\end{array}$ & \\
\hline Surgery $+R T$ & $9,898(2.7)$ & $9,405(2.7)$ & $293(3.6)$ & $25(3.2)$ & $49(3.8)$ & $11(3.7)$ & $115(3.8)$ & \\
\hline $\begin{array}{l}\text { Surgery }+ \\
\text { Unknown RT }\end{array}$ & $2,154(0.6)$ & $2,098(0.6)$ & $33(0.4)$ & $3(0.4)$ & $4(0.3)$ & $2(0.7)$ & $14(0.5)$ & \\
\hline Unknown & $5,264(1.4)$ & $5,093(1.4)$ & $86(1.1)$ & $12(1.5)$ & $21(1.6)$ & $10(3.3)$ & $42(1.4)$ & \\
\hline $\begin{array}{l}\text { Unknown Surgery } \\
+R T\end{array}$ & $203(0.1)$ & $198(0.1)$ & $2(0.0)$ & $0(0.0)$ & $1(0.1)$ & $1(0.3)$ & $1(0.0)$ & \\
\hline Grade $^{a}$ & & & & & & & & $<0.001$ \\
\hline $\begin{array}{l}\text { Well } \\
\text { differentiated }\end{array}$ & $3,720(1.0)$ & $3,510(1.0)$ & $118(1.5)$ & $17(2.2)$ & 25 (1.9) & $4(1.3)$ & $46(1.5)$ & \\
\hline $\begin{array}{l}\text { Moderately } \\
\text { differentiated }\end{array}$ & $\begin{array}{c}165,589 \\
(45.2)\end{array}$ & $\begin{array}{c}159,535 \\
(45.2)\end{array}$ & $3,524(43.6)$ & $359(45.6)$ & $603(47.0)$ & $147(49.0)$ & $\begin{array}{l}1,421 \\
(47.0)\end{array}$ & \\
\hline $\begin{array}{l}\text { Poorly } \\
\text { differentiated }\end{array}$ & $\begin{array}{c}187,875 \\
(51.3)\end{array}$ & $\begin{array}{c}181,012 \\
(51.3)\end{array}$ & $4,247(52.6)$ & $387(49.1)$ & $618(48.1)$ & $137(45.7)$ & $\begin{array}{l}1,474 \\
(48.8)\end{array}$ & \\
\hline Undifferentiated & $755(0.2)$ & $732(0.2)$ & $12(0.1)$ & $2(0.3)$ & $3(0.2)$ & $1(0.3)$ & $5(0.2)$ & \\
\hline Unknown & $8,417(2.3)$ & $8,097(2.3)$ & $176(2.2)$ & $23(2.9)$ & $35(2.7)$ & $11(3.7)$ & $75(2.5)$ & \\
\hline
\end{tabular}

Abbreviations: $\mathrm{SES}=$ socioeconomic status; $\mathrm{RT}=$ radiotherapy.

P-values were obtained from Kruskal-Wallis test for continuous variable and Chi-square test for categorical variables.

${ }^{a}$ Well differentiated, moderately differentiated, and poorly differentiated histologic grading correlate with Gleason scores $2-4,5-6$, and 7-10, respectively. 
showed no significance. In the MVA, Puerto Rican (HR= $1.50,95 \%$ CI 1.12 to $1.96, p=0.006)$ men continued to have significantly higher PCSM compared to NHB men, indicating that this group is at greater risk for PCSM. Mexican $(\mathrm{HR}=1.08,95 \% \mathrm{CI} 0.93$ to $1.25, p=0.307)$ men did not show significantly different risk compared to NHB men.

\section{DISCUSSION}

Historically, the identification of Hispanic/ Latino individuals has primarily been based on political and geographic concepts [10]. Within this group, are individuals varying by language, ancestral mixture, and cultural practices [22]. Due to the unique bond shared amongst these populations and for other sociopolitical reasons, these groups have historically been aggregated. As thoughtfully outlined by Kaplan et al [7], three challenges exist when writing about race and/or ethnicity: 1) accounting for limitations of data, 2) distinguishing between race and/or ethnicity as a risk factor or as a risk marker, and 3) writing about race and/or ethnicity in a way that does not stigmatize nor create a we/they dichotomy between health professionals and minority populations. For this reason, the American Medical Association has recommended to use more descriptive terms for Hispanic/ Latino individuals (e.g. Mexican American, Puerto Rican, or Cuban American), when possible [11, 23]. However, our use of the term ethnic heterogeneity is not intended to discredit the use of ethnicity or its use in identifying populations not receiving medical services, but instead to

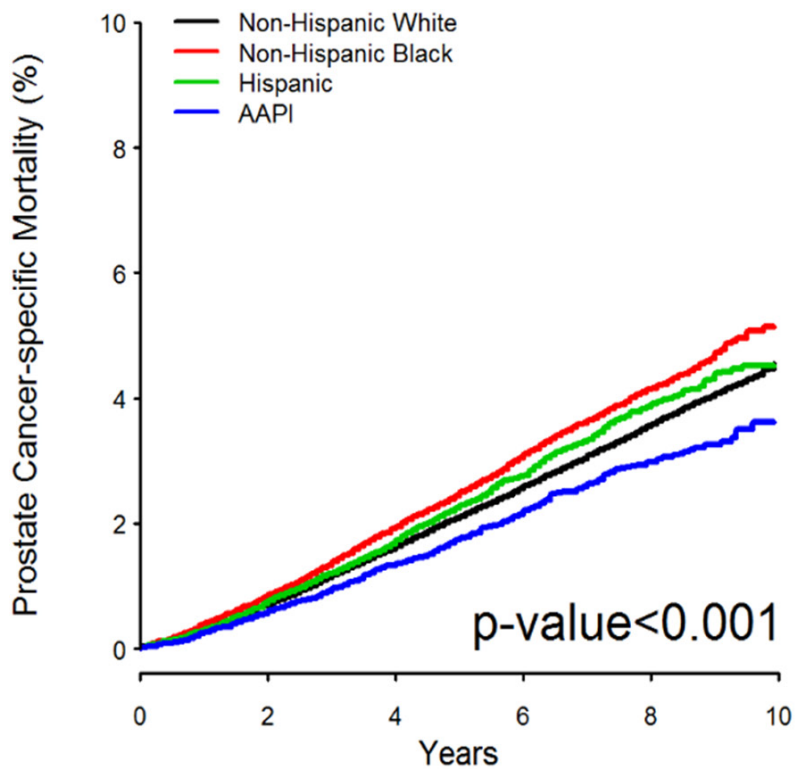

create a broader understanding of sociocultural labels and the significant clinical implications they may have on our patients.

To our knowledge, this is the first report using a national database to explore ethnic heterogeneity of Hispanic/Latino men in PCa outcomes. One Surveillance, Epidemiology, and End Results (SEER)-Medicare study included Hispanic/Latino men, but showed improved $\mathrm{PCa}$ related outcomes compared to NHW and NHB patients [15]. A limitation of this study was that they had a relatively small Hispanic/Latino cohort that was 66 years or older, which may not be generalizable to younger men. Two all site cancer studies that included Hispanic/Latino subgroups were conducted with state of Florida cohorts $[20,21]$, where percent distribution of PCa incidence is predominantly Cuban (39\%), with fewer Puerto Rican (7\%), Mexican (2\%), South and Central American (7\%), other Hispanic/Latino (10\%), and Hispanic/Latino, NOS $(35 \%)$ men [24]. While these state-specific demographics would be difficult to generalize nationally, the SEER database is more representative of the national picture of racial and ethnic diversity. In a recent review article, Stern et al [25] discuss reports of lower PCa incidence rates for Mexican Americans and similar or slightly higher rates for Cuban Americans and Puerto Ricans compared to NHW men $[20,26,27]$. Additionally, they discuss several other reports demonstrating the influence of SES deprivation [28], U.S. vs. foreign-born status [16], and living in predominantly Hispanic/Latino enclaves [16, 29] on mortality rates. By distinguishing between Hispanic/ Latino subgroups in national populations, we are better

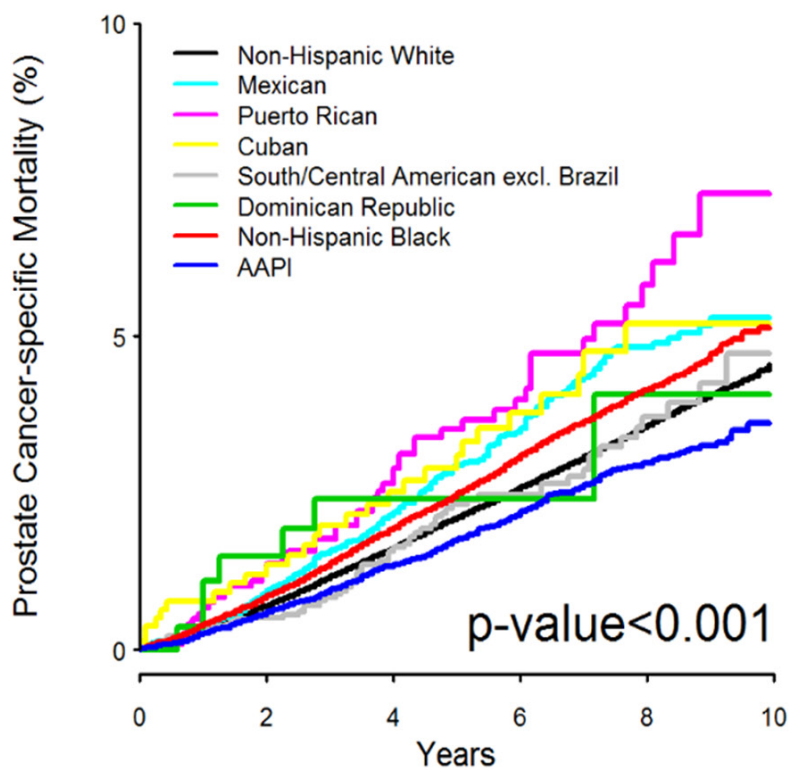

Figure 1: Cumulative incidence of prostate cancer-specific mortality in years divided by race and/or ethnicity: (left) Non-Hispanic White (black), Non-Hispanic Black (red), Hispanic/Latino (green), and Asian American/Pacific Islander (blue); (right) Non-Hispanic White (black), non-Hispanic Black (red), Asian American/Pacific Islander (blue), Mexican American (cyan), Puerto Rican (purple), Cuban (yellow), South or Central American excluding Brazil (grey), and Dominican Republic (green). 
Table 3. Fine-gray competing risks regression analyses for PCSM by race and/or ethnicity using Non-Hispanic White cohort as the reference

\begin{tabular}{|c|c|c|c|c|}
\hline \multirow{2}{*}{ Variable } & \multicolumn{2}{|c|}{ Univariable Analyses } & \multicolumn{2}{|c|}{ Multivariable Analyses ${ }^{a}$} \\
\hline & HR (95\% CI) & p-value & HR (95\% CI) & p-value \\
\hline Non-Hispanic White & 1.0 (reference) & - & 1.0 (reference) & - \\
\hline Non-Hispanic Black & $1.18(1.12,1.24)$ & $<0.001$ & $1.15(1.09,1.22)$ & $<0.001$ \\
\hline Hispanic/Latino $^{b}$ & $1.07(1.00,1.15)$ & 0.055 & $1.02(0.95,1.10)$ & 0.534 \\
\hline$A A P I$ & $0.83(0.75,0.92)$ & $<0.001$ & $0.74(0.67,0.82)$ & $<0.001$ \\
\hline Non-Hispanic White & 1.0 (reference) & - & 1.0 (reference) & - \\
\hline Mexican & $1.34(1.17,1.53)$ & $<0.001$ & $1.21(1.05,1.39)$ & 0.008 \\
\hline Puerto Rican & $1.70(1.28,2.25)$ & $<0.001$ & $1.71(1.29,2.26)$ & $<0.001$ \\
\hline Cuban & $1.45(0.99,2.12)$ & 0.054 & $1.21(0.83,1.78)$ & 0.328 \\
\hline $\begin{array}{l}\text { South or Central } \\
\text { American }\end{array}$ & $0.99(0.77,1.28)$ & 0.957 & $1.11(0.86,1.43)$ & 0.437 \\
\hline Dominican Republic & $1.23(0.58,2.59)$ & 0.586 & $1.60(0.77,3.33)$ & 0.211 \\
\hline
\end{tabular}

Abbreviations: $\mathrm{HR}=$ hazard ratio; AAPI = Asian American/Pacific Islander.

HR and p-value were obtained using Fine-Gray competing risks regression model.

${ }^{a}$ Multivariable analysis was conducted with competing risks regression analysis adjusting for age at diagnosis, disease stage summary, grade, treatment type, SES composite score, insurance status, marital status, and residence type.

${ }^{b}$ Represents all Hispanic/Latino individuals from the study, including those reported as Hispanic/Latino, NOS.

Table 4: Fine-gray competing risks regression analyses for PCSM by race and/or ethnicity using Non-Hispanic Black cohort as the reference

\begin{tabular}{|c|c|c|c|c|}
\hline \multirow{2}{*}{ Variable } & \multicolumn{2}{|c|}{ Univariable Analyses } & \multicolumn{2}{|c|}{ Multivariable Analyses ${ }^{a}$} \\
\hline & HR $(95 \%$ CI) & p-value & HR (95\% CI) & p-value \\
\hline Non-Hispanic Black & 1.0 (reference) & - & 1.0 (reference) & - \\
\hline Non-Hispanic White & $0.85(0.81,0.90)$ & $<0.001$ & $0.87(0.82,0.92)$ & $<0.001$ \\
\hline Hispanic/Latino ${ }^{b}$ & $0.91(0.84,0.99)$ & 0.022 & $0.89(0.82,0.96)$ & 0.005 \\
\hline$A A P I$ & $0.71(0.63,0.79)$ & $<0.001$ & $0.64(0.57,0.72)$ & $<0.001$ \\
\hline Non-Hispanic Black & 1.0 (reference) & - & 1.0 (reference) & - \\
\hline Mexican & $1.14(0.99,1.31)$ & 0.065 & $1.08(0.93,1.25)$ & 0.307 \\
\hline Puerto Rican & $1.45(1.09,1.92)$ & 0.011 & $1.50(1.12 ., 1.96)$ & 0.006 \\
\hline Cuban & $1.24(0.85,1.81)$ & 0.274 & $1.13(0.74,1.60)$ & 0.679 \\
\hline $\begin{array}{l}\text { South or Central } \\
\text { American }\end{array}$ & $0.84(0.66,1.09)$ & 0.189 & $0.93(0.73,1.23)$ & 0.676 \\
\hline Dominican Republic & $1.04(0.49,2.20)$ & 0.915 & $1.18(0.64,2.79)$ & 0.436 \\
\hline
\end{tabular}

Abbreviations: $\mathrm{HR}=$ hazard ratio; AAPI = Asian American/Pacific Islander.

HR and p-value were obtained using Fine-Gray competing risks regression model.

aMultivariable analysis was conducted with competing risks regression analysis adjusting for age at diagnosis, disease stage summary, grade, treatment type, SES composite score, insurance status, marital status, and residence type.

${ }^{b}$ Represents all Hispanic/Latino individuals from the study, including those reported as Hispanic/Latino, NOS. 
poised to understand observed variability in disease outcomes and to address associated risk factors and risk conditions in order to assist clinical and public health decision-making.

Our findings recognize two population subgroups within the Hispanic/Latino community at similar or greater risk for PCSM than NHB men. Adjusting for the aforementioned covariates, Puerto Rican men had significantly higher PCSM compared to NHW and NHB groups. Mexican men had significantly higher PCSM compared to NHW men and showed no difference compared to NHB men. In the largest U.S. ethnic group, Mexicans comprise the majority with about 35 million people and Puerto Ricans are the second largest group with about 5 million people living in the mainland [12]. In regards to $\mathrm{PCa}$ disparities, an emphasis has been placed on NHB men due to higher rates of adverse outcomes compared to NHW men. The discovery that Puerto Rican men have a greater risk and Mexican American men have similar risk for PCSM compared to NHB men calls for a deeper understanding of the etiology of these disparities. Likely due to power limitations, we are unable to draw conclusions regarding Cuban and Dominican men who did not show statistical significance in regression models of PCSM.

The high rate of PCSM mortality in NHB men has been attributed to both socioeconomic [30] and biological [31] factors, a better understanding of which will contribute to improving outcomes. Efforts made to understand these factors have resulted in proposals for race-based risk classifiers when counseling patients on management strategies [32]. Although the United States Preventative Services Task Force recommends individualized decision-making about PSA screening after discussion of pros and cons with a clinician, they do recognize the higher rates of aggressive cancer in NHB men [33]. The American Cancer Society recommends that all men with average risk and older than 50 years should receive PSA screening information to make an informed decision with their provider about being tested. They recommend this information be offered to $\mathrm{NHB}$ men and those with a family history of PCa at 45 years of age and men with more than one first-degree relative who had $\mathrm{PCa}$ at an early age should be offered such information at 40 years of age [34]. Variations in screening guideline recommendations are a reflection of the impact made by the identification and further characterization of these atrisk men. Although PSA is a suboptimal screening tool that will likely be enhanced/substituted over time (i.e. PCA3, $\mathrm{PHI}, 4 \mathrm{~K}$ score), our findings indicate that consideration be given to the ethnic background of Hispanic/Latino men.

A strength of our study is the use of a contemporary population-based cancer registry containing a large, diverse cohort. However, the SEER database has potential limitations. By using the NHIA recode for ethnicity, there is potential for U.S. born Hispanic/Latino men to have missing country of origin or ancestry data due to lack of affiliation with Hispanic/Latino identity or no specific information is provided [35]. For this reason, U.S. born Hispanic/Latino men identified by surname only are thus labeled as Hispanic/Latino, NOS. In our cohort, less than $50 \%$ of the Hispanic/Latino men were identified to belong to a specific Hispanic/Latino subgroup, which could have weakened the power of subgroup analyses. The inability to further specify the region of origin or ancestry within the South or Central American group was also a source of limitation due to the potential for within-group differences. Though Cuban, South or Central American, and Dominican subgroups were not found to be significantly different than NHW or NHB men, the cumulative incidence plots (Figure 1B) suggest diversity in these populations requiring further investigation. Beginning with the 2014 submission, SEER no longer provides birthplace data due to disproportional documentation rates in living vs. deceased cases [36-38]. With immigrants constituting a significant portion of the Hispanic/Latino community, the absence of this information limits our ability to account for potential differences between U.S. and foreign-born Hispanic/Latino men.

Another potential limitation in our study is the use of poverty, education, and residence type information collected at the county level, not the individual or census tract level. While several publications have described the benefits of monitoring inequalities through census tract level data [39], this information is not available in SEER. PSA levels were not made available on the 2015 SEER data release due to questionable accuracy, thus not included in this analysis [19]. According to guidelines from the National Comprehensive Cancer Network, patients diagnosed with metastatic $\mathrm{PCa}$ are primarily treated with chemotherapy and/or hormone therapy, but SEER does not provide such data. For this reason, patients with metastatic disease were excluded to eliminate these potential confounders when adjusting for treatment type in the MVA. Lastly, a comorbidity index is only available in the SEER-Medicare linked data. We chose to use the entire SEER dataset to maximize the power in the examination of Hispanic/Latino subgroups. The use of cancer-specific mortality as an endpoint minimizes the influence of comorbidity as a confounder. The findings that such disparities in PCSM exist should be recognized, regardless of potential influences.

In summary, our data indicate that ethnic heterogeneity amongst Hispanic/Latino populations in the United States significantly contributes to PCSM, which should be considered in guidelines for clinical practice. Two distinct subgroups had PCSM rates that were higher than all other racial and/or ethnic groups when compared to NHW men. Compared to NHB men, PCSM was significantly worse in Puerto Rican men and showed no difference in Mexican men. As appreciated in the NHB population, this type of information drives screening policy recommendations and affects management 
recommendations, including active surveillance. Further investigation into potentially contributing biological, socioeconomic, and/or sociocultural factors unique to certain Hispanic/Latino subgroups is warranted.

\section{MATERIALS AND METHODS}

PCa incidence and outcome data was obtained from 18 registries of the November 2015 submission offered by the SEER program of the National Cancer Institute, which includes about $30 \%$ of the U.S. population. Analyses of these data are considered nonhuman subjects research by the U.S. Department of Health and Human Services' Office for Human Research Protection because it is deidentified and publicly available, which does not require institutional review board approval.

\section{Definitions}

Since race and ethnicity are constantly evolving concepts and used ubiquitously in medical literature [40], the terms are defined accordingly. Race is a category of humankind that shares certain distinctive physical traits [41]. Ethnicity refers to groups of people classed according to common racial, national, tribal, religious, linguistic, or cultural origin or background [41]. According to the U.S. Census Bureau, Hispanic or Latino refers to those who identify with being Mexican, Puerto Rican, Cuban, Dominican, Central or South American, or from a country of Spanish-speaking origin [42]. However, others have stated that the term Latino includes any person with origins from Latin America, including Brazil [10]. Since the SEER database does not include Brazilian Americans in their Hispanic origin recode, we use Hispanic/Latino to denote all U.S. persons whose origins can be traced to the Spanish-speaking regions of Latin America.

\section{National hispanic identification algorithm}

From the SEER database, we used the National Hispanic Identification Algorithm (NHIA) origin recode designed by the North American Association of Central Cancer Registries (NAACCR) for ethnicity data [43]. This algorithm evaluates surname, race, ethnicity selfidentification, and birthplace to assign a code of nonHispanic, a specific Hispanic/Latino subgroup, or Hispanic/Latino, not otherwise specified (NOS). Only those who self-identify or have heavily Hispanic surnames and are not of AAPI racial background are concluded to have Hispanic/Latino ethnicity. The successful use of this recode has been exhibited in similar publications [44].

\section{Patient population}

Men diagnosed with PCa from 2000-2013 (n= 791,234) were extracted from the SEER database. All men with Mx/M1 staging or distant disease $(n=285,897)$ were excluded to avoid any confounding variables related to chemotherapy or hormone therapy, which are not documented in the SEER program. We identified 505,337 men with local-regional PCa from 2000-2013 (Supplementary Figure 1). Our final cohort $(n=486,865)$ compared the following racial and/or ethnic groups: NHW $(n=352,886)$, NHB $(n=70,983)$, Hispanic/Latino $(n=40,462)$, and AAPI $(n=22,534)$. Other racial/ethnic groups $(n=18,472)$ were not included in the analysis. Additionally, we compared Hispanic/Latino subgroups: Mexican $(n=8,077)$, Puerto Rican $(n=1,284)$, South or Central American $(n=3,021)$, Cuban $(n=788)$, and Dominican $(n=300)$. Any patient identified as Hispanic/ Latino, NOS $(n=26,992)$ without specific country of origin or ancestry information was excluded from the Hispanic/Latino subgroup analysis.

\section{Study variables}

Variables that were evaluated include race and/or ethnicity (NHW, NHB, Hispanic/Latino, or AAPI), ethnic subgroups (Mexican, Puerto Rican, Cuban, Dominican, or South or Central American excluding Brazil), age at diagnosis, TNM staging, disease stage summary (localized vs. regional disease), grade, treatment type (none vs. surgery only vs. radiation treatment [RT] only vs. surgery + RT vs. surgery + unknown RT vs. unknown surgery + RT vs. unknown), SES composite score, insurance status (uninsured vs. any Medicaid vs. insured vs. unknown), marital status (married vs. others vs. unknown), and residence type (rural vs. urban).

\section{Socioeconomic status composite score}

We collected three county level variables representative of SES: 1) education: percent of adults at age 25 years or older with less than a $12^{\text {th }}$ grade education; 2) poverty: percent of people living below the poverty line; and 3) income: median annual household income. Each individual variable was standardized into values ranging from 0 to 1 or low SES to high SES, respectively. All three variables were then added and the sum for each case was equally weighted to create an SES composite score based on previously published methodologies to provide a multidimensional standardized measure of socioeconomic conditions [45].

\section{Statistical analyses}

The cumulative incidence of PCSM for all racial and/ or ethnic groups was examined generally (Figure 1) and with categorical age groups: $<65$ years old (Supplementary Figure 2 ) and $\geq 65$ years old (Supplementary Figure 3 ). In the supplement, patient characteristic tables were provided for men $<65$ years old (Supplementary Tables 1 and 2) and $\geq 65$ years old (Supplementary Tables 3 and 4 ). Fiveyear cumulative incidence rates for PCSM, non-PCSM, 
and all-cause mortality are reported in Supplementary Table 5. We used Fine-Gray competing risks regression for the UVA and MVA [46]. In the MVA, we included age at diagnosis, disease stage summary, grade, treatment type, SES composite score, insurance status, marital status, and residence type for adjustment. In separate UVA and MVA, NHW and NHB were used as reference groups. Median follow-up among surviving patients was 50.4 months (0-119 months). All statistical tests were two-sided, and p-values less than 0.05 were considered statistically significant. Analyses involving competing risks were performed using R software (http://www.r-project.org) and all other analyses were carried out using SAS (SAS Institute, Cary, NC).

\section{Abbreviations}

$\mathrm{PCa}=$ prostate cancer

PCSM $=$ prostate cancer specific mortality

NHW $=$ Non-Hispanic White

$\mathrm{NHB}=$ Non-Hispanic Black

AAPI $=$ Asian American/Pacific Islander

$\mathrm{UVA}=$ univariable analysis

MVA $=$ multivariable analysis

SES $=$ socioeconomic status

Results

SEER = Surveillance, Epidemiology, and End

NHIA $=$ National Hispanic Identification Algorithm

NAACCR $=$ North American Association of Central

Cancer Registries

NOS $=$ not otherwise specified

\section{Author contributions}

Study conception: All authors

Methodology: All authors

Computation: Kwon

Formal analysis: Kwon

Investigation: data/evidence collection: Chinea,

Patel, Kwon, Pollack

Resources: Chinea, Patel, Lopez, Lamichhane

Data curation: Chinea, Kwon

Writing/manuscript preparation: writing the initial draft: Chinea, Patel

Writing/manuscript preparation: critical review, commentary, or revision: All authors

Writing/manuscript preparation: visualization/data presentation: Chinea, Patel, Kwon, Pollack

Supervision: Chinea, Patel, Pollack

Project administration: Lopez, Lamichhane

Funding acquisition: Pollack, Kobetz

\section{ACKNOWLEDGMENTS}

We acknowledge the efforts of the National Cancer Institute and the SEER Program tumor registries in the creation of the SEER database.

\section{CONFLICTS OF INTEREST}

All authors had no conflicts of interest related to this study.

\section{FINANCIAL SUPPORT}

This work was supported by the National Institutes of Health (R01CA189295 and R01CA190105 to A.P., 1U54CA153705-01 and 1R01CA183612-01 to E.K.). The content is solely the responsibility of the authors and does not necessarily represent the official views of the NIH.

\section{REFERENCES}

1. Ries L, Hankey B, Kosary C, Harras A, Edwards B. SEER Cancer Statistics Review, 1973-1991: Tables and Graphs, National Cancer Institute. NIH Publication no. 94-2789. 1994.

2. Mahal BA, Ziehr DR, Aizer AA, Hyatt AS, Sammon JD, Schmid M, Choueiri TK, Hu JC, Sweeney CJ, Beard CJ, D'Amico AV, Martin NE, Lathan C, et al. Getting back to equal: The influence of insurance status on racial disparities in the treatment of African American men with high-risk prostate cancer. Urologic oncology. 2014; 32:1285-1291.

3. Mahal BA, Aizer AA, Ziehr DR, Hyatt AS, Sammon JD, Schmid M, Choueiri TK, Hu JC, Sweeney CJ, Beard CJ, D'Amico AV, Martin NE, Kim SP, et al. Trends in disparate treatment of African American men with localized prostate cancer across National Comprehensive Cancer Network risk groups. Urology. 2014; 84:386-392.

4. Kryvenko ON, Balise R, Soodana Prakash N, Epstein JI. African-American Men with Gleason Score 3+3=6 Prostate Cancer Produce Less Prostate Specific Antigen than Caucasian Men: A Potential Impact on Active Surveillance. J Urol. 2016; 195:301-306.

5. Sundi D, Kryvenko ON, Carter HB, Ross AE, Epstein JI, Schaeffer EM. Pathological examination of radical prostatectomy specimens in men with very low risk disease at biopsy reveals distinct zonal distribution of cancer in black American men. J Urol. 2014; 191:60-67.

6. LaVeist TA. Beyond dummy variables and sample selection: what health services researchers ought to know about race as a variable. Health Serv Res. 1994; 29:1-16.

7. Kaplan JB, Bennett $T$. Use of race and ethnicity in biomedical publication. Jama. 2003; 289:2709-2716.

8. Ennis S, Rios-Vargas M, Albert NG. (2011). The Hispanic Population: 2010. 2010 Census Briefs: U.S. Census.

9. Siegel RL, Miller KD, Jemal A. Cancer statistics, 2016. CA Cancer J Clin. 2016; 66:7-30.

10. Hayes-Bautista DE, Chapa J. Latino terminology: conceptual bases for standardized terminology. Am J Public Health. 1987; 77:61-68.

11. Trevino FM. Standardized terminology for hispanic populations. Am J Public Health. 1987; 77:69-72. 
12. Motel S, Patten E. (2012). The 10 Largest Hispanic Origin Groups: Characteristics, Rankings, Top Counties/Source: Pew Hispanic Center tabulations of the 2010 ACS. In: M R, ed. Hispanic Trends: Pew Research Center.

13. Arauz A, Romano JG, Ruiz-Franco A, Shang T, Dong C, Rundek T, Koch S, Hernandez-Curiel B, Pacheco J, Rojas P, Ruiz-Navarro F, Katsnelson M, Sacco RL. Differences in lipid profiles in two Hispanic ischemic stroke populations. International journal of stroke. 2014; 9:394-399.

14. Chao GF, Krishna N, Aizer AA, Dalela D, Hanske J, Li H, Meyer CP, Kim SP, Mahal BA, Reznor G, Schmid M, Choueiri TK, Nguyen PL, et al. Asian Americans and prostate cancer: A nationwide population-based analysis. Urologic oncology. 2015; 34:233.e7-15.

15. Chhatre S, Bruce Malkowicz S, Sanford Schwartz J, Jayadevappa R. Understanding the Racial and Ethnic Differences in Cost and Mortality Among Advanced Stage Prostate Cancer Patients (STROBE). Medicine. 2015; 94:e1353.

16. Schupp CW, Press DJ, Gomez SL. Immigration factors and prostate cancer survival among Hispanic men in California: does neighborhood matter? Cancer. 2014; 120:1401-1408.

17. Ziehr DR, Mahal BA, Aizer AA, Hyatt AS, Beard CJ, D'Amico AV, Choueiri TK, Elfiky A, Lathan CS, Martin NE, Sweeney CJ, Trinh QD, Nguyen PL. Income inequality and treatment of African American men with high-risk prostate cancer. Urologic oncology. 2015; 33:18 e17-13.

18. Parker PM, Rice KR, Sterbis JR, Chen Y, Cullen J, McLeod DG, Brassell SA. Prostate cancer in men less than the age of 50: a comparison of race and outcomes. Urology. 2011; $78: 110-115$.

19. Freeman VL, Ricardo AC, Campbell RT, Barrett RE, Warnecke RB. Association of census tract-level socioeconomic status with disparities in prostate cancerspecific survival. Cancer epidemiology, biomarkers \& prevention. 2011; 20:2150-2159.

20. Pinheiro PS, Sherman RL, Trapido EJ, Fleming LE, Huang Y, Gomez-Marin O, Lee D. Cancer incidence in first generation U.S. Hispanics: Cubans, Mexicans, Puerto Ricans, and new Latinos. Cancer epidemiology, biomarkers \& prevention. 2009; 18:2162-2169.

21. Martinez-Tyson D, Pathak EB, Soler-Vila H, Flores AM. Looking under the Hispanic umbrella: cancer mortality among Cubans, Mexicans, Puerto Ricans and other Hispanics in Florida. Journal of immigrant and minority health. 2009; 11:249-257.

22. Gonzalez Burchard E, Borrell LN, Choudhry S, Naqvi M, Tsai HJ, Rodriguez-Santana JR, Chapela R, Rogers SD, Mei R, Rodriguez-Cintron W, Arena JF, Kittles R, PerezStable EJ, et al. Latino populations: a unique opportunity for the study of race, genetics, and social environment in epidemiological research. Am J Public Health. 2005; 95:2161-2168.

23. Young R. (2007). Race/Ethnicity. AMA Manual of Style: A Guide for Authors and Editors: Oxford University Press.
24. Hernandez MN, Fleming LE, MacKinnon JA, Lee DJ. (2010). Cancer in Florida Hispanics 1989-2006. Miami: Florida Cancer Data System.

25. Stern MC, Fejerman L, Das R, Setiawan WV, Cruz-Correa MR, Perez-Stable EJ, Figueiredo JC. Variability in Cancer Risk and Outcomes Within US Latinos by National Origin and Genetic Ancestry. Cancer Epidemiology Reports. 2016; 3:181-190.

26. Howe HL, Lake A, Schymura MJ, Edwards BK. Indirect method to estimate specific Hispanic group cancer rates. Cancer Causes Control. 2009; 20:1215-1226.

27. Howe HL, Wu X, Ries LA, Cokkinides V, Ahmed F, Jemal A, Miller B, Williams M, Ward E, Wingo PA, Ramirez A, Edwards BK. Annual report to the nation on the status of cancer, 1975-2003, featuring cancer among U.S. Hispanic/ Latino populations. Cancer. 2006; 107:1711-1742.

28. Philips BU Jr, Belasco E, Markides KS, Gong G. Socioeconomic deprivation as a determinant of cancer mortality and the Hispanic paradox in Texas, USA. Int $\mathbf{J}$ Equity Health. 2013; 12:26.

29. Soto-Salgado M, Suarez E, Torres-Cintron M, Pettaway CA, Colon V, Ortiz AP. Prostate cancer incidence and mortality among Puerto Ricans: an updated analysis comparing men in Puerto Rico with US racial/ethnic groups. P R Health Sci J. 2012; 31:107-113.

30. Mahal BA, Aizer AA, Ziehr DR, Hyatt AS, LagoHernandez C, Chen YW, Choueiri TK, Hu JC, Sweeney CJ, Beard CJ, D'Amico AV, Martin NE, Trinh QD, Nguyen PL. The association between insurance status and prostate cancer outcomes: implications for the Affordable Care Act. Prostate Cancer Prostatic Dis. 2014; 17:273-279.

31. Powell IJ, Bock CH, Ruterbusch JJ, Sakr W. Evidence supports a faster growth rate and/or earlier transformation to clinically significant prostate cancer in black than in white American men, and influences racial progression and mortality disparity. J Urol. 2010; 183:1792-1796.

32. Sundi D, Ross AE, Humphreys EB, Han M, Partin AW, Carter HB, Schaeffer EM. African American men with very low-risk prostate cancer exhibit adverse oncologic outcomes after radical prostatectomy: should active surveillance still be an option for them? Journal of clinical oncology. 2013; 31:2991-2997.

33. (2017). Draft Recommendation Statement: Prostate Cancer: Screening. U.S. Preventative Services Task Force.

34. Wolf AM, Wender RC, Etzioni RB, Thompson IM, D'Amico AV, Volk RJ, Brooks DD, Dash C, Guessous I, Andrews K, DeSantis C, Smith RA, American Cancer Society Prostate Cancer Advisory C. American Cancer Society guideline for the early detection of prostate cancer: update 2010. CA Cancer J Clin. 2010; 60:70-98.

35. Boscoe FP, Schymura MJ, Zhang X, Kramer RA. Heuristic algorithms for assigning Hispanic ethnicity. PLoS One. 2013; 8:e55689.

36. Gomez SL, Glaser SL, Kelsey JL, Lee MM. Bias in completeness of birthplace data for Asian groups in a 
population-based cancer registry (United States). Cancer Causes Control. 2004; 15:243-253.

37. Montealegre JR, Zhou R, Amirian ES, Scheurer ME. Uncovering nativity disparities in cancer patterns: Multiple imputation strategy to handle missing nativity data in the Surveillance, Epidemiology, and End Results data file. Cancer. 2014; 120:1203-1211.

38. Pinheiro PS, Bungum TJ, Jin H. Limitations in the imputation strategy to handle missing nativity data in the Surveillance, Epidemiology, and End Results program. Cancer. 2014; 120:3261-3262.

39. Krieger N, Chen JT, Waterman PD, Rehkopf DH, Subramanian SV. Painting a truer picture of US socioeconomic and racial/ethnic health inequalities: the Public Health Disparities Geocoding Project. Am J Public Health. 2005; 95:312-323.

40. Winker MA. Measuring race and ethnicity: why and how? Jama. 2004; 292:1612-1614.

41. Merriam-Webster Inc. (2011). Merriam-Webster's collegiate dictionary. (Springfield, Mass.: Merriam-Webster).
42. (2010). Hispanic Origin. Population Estimates Program (PEP): U.S. Bureau of the Census).

43. (2011). NAACCR Guideline for Enhancing Hispanic/ Latino Identification: Revised NAACCR Hispanic/Latino Identificaton Algorithm [xNHIA v2.2.1]. In: Group NRaEW, ed. (Springfield (IL): North American Association of Central Cancer Registries.

44. Iqbal J, Ginsburg O, Rochon PA, Sun P, Narod SA. Differences in breast cancer stage at diagnosis and cancerspecific survival by race and ethnicity in the United States. Jama. 2015; 313:165-173.

45. Robert SA, Strombom I, Trentham-Dietz A, Hampton JM, McElroy JA, Newcomb PA, Remington PL. Socioeconomic risk factors for breast cancer: distinguishing individual- and community-level effects. Epidemiology. 2004; 15:442-450.

46. Fine JP, Gray RJ. A proportional hazards model for the subdistribution of a competing risk. J Am Stat Assoc. 1999; 94:496-509. 